\title{
Repoblamiento, actividades productivas y percepción del riesgo en Corral, 50 años después del tsunami de $1960^{1}$
}

\author{
Patricia Gutiérrez ${ }^{2}$, Fernando Peña-Cortés ${ }^{3}$ y Marco Cisternas ${ }^{4}$
}

\begin{abstract}
RESUMEN
El análisis de la evolución del repoblamiento y uso de áreas afectadas postsunami, cobra importancia después de los últimos eventos catastróficos de Chile y Japón. Sin embargo, por tratarse de eventos recientes, se carece de una perspectiva de largo término. El presente estudio explora la evolución del repoblamiento y actividades productivas de un poblado ícono de la destrucción generada por una catástrofe ocurrida en Chile, hace medio siglo: el terremoto y tsunami de 1960 en Corral. El estudio se desarrolló a través de tres métodos: i) análisis de fotografías aéreas antiguas, ii) encuestas a sobrevivientes, y iii) análisis de los Instrumentos de Planificación Territorial (IPTs) entre 1960 y 2009. Los resultados indican que las áreas inundadas en 1960 fueron nuevamente repobladas y que la percepción actual frente al riesgo es baja. Por otro lado, se detectó la falta de carácter normativo de esta temática en los IPTs vigentes a nivel comunal.
\end{abstract}

Palabras clave: Tsunami 1960, desastre, gestión del riesgo.

\begin{abstract}
Post-tsunami analysis of the evolution of resettlement and land use changes in affected areas has become particularly important following the latest catastrophic events in Chile and Japan. Nevertheless, due to the recent nature of these events there is a lack of long-term perspectives. The present study explores the evolution of resettlement and productive activities within a town representative of the destruction generated by the catastrophe that occurred in Chile half a century ago: the earthquake and tsunami of 1960 in Corral. The study used three main methods: i) analysis of old aerial photographs, ii) interviews with survivors, and iii) analysis of Instruments of Territorial Planning (IPTs) between 1960 and 2009. The results show the 1960 inundated areas were resettled again and that the current risk awareness is low. Additionally, it was detected that there is a lack of regulation regarding this topic in the IPTs in existence at a local level.
\end{abstract}

Key words: tsunami 1960, disaster, hazard management.

\footnotetext{
Los autores agradecen el apoyo de los proyectos FONDECYT 1110798, FONDEF TIC-EDU TE10I003 y DGIPUCT $\mathrm{N}^{\circ} \mathrm{CD}$ 2010-01. Asimismo, al equipo del Laboratorio de Planificación Territorial de la Universidad Católica de Temuco, a la Secretaría Ministerial de Vivienda y Urbanismo de la Región de Los Ríos, y especialmente a Jimmy Pincheira por su colaboración en la revisión del texto. Artículo recibido el 29 de agosto de 2012, aceptado el 26 de septiembre de 2012 y corregido el 4 de octubre de 2012.
}

2 Programa de Magíster en Planificación y Gestión Territorial/ Laboratorio de Planificación Territorial, Universidad Católica de Temuco (Chile). E-mail: pgutierr@uct.cl

3 Laboratorio de Planificación Territorial, Núcleo de Investigación en Estudios Ambientales, Universidad Católica de Temuco (Chile).E-mail: fpena@uct.cl

4 Escuela de Ciencias del Mar, Pontificia Universidad Católica de Valparaíso (Chile).

E-mail: marco.cisternas@ucv.cl 
Chile tiene $4.200 \mathrm{~km}$ de costa localizada sobre una de las zonas de subducción sísmicamente más activas del planeta. Uno de los principales efectos de los grandes sismos de subducción son los tsunamis (Caicedo et al., 2001). Los tsunamis son fenómenos periódicos que ocurren en el mar y que inundan la costa. Son generados por un disturbio que impulsa y desplaza verticalmente la columna de agua originando un tren de ondas de largo período. Se propagan a gran velocidad en todas direcciones desde su origen, y que al aproximarse a la costa alcanzan alturas de grandes proporciones. Descargan su energía con gran poder, infligiendo una vasta destrucción y modificaciones en el sistema costero (Mascarenhas, 2006; Sonak et al., 2008; Mascarenhas \& Jayakumar, 2008). Las principales modificaciones se asocian a cambios en la batimetría, modificaciones geomorfológicas, como erosión o acreción a lo largo de la costa, salinización de tierras agrícolas y de agua dulce, depositación de materiales orgánicos y basura en el fondo del mar e impactos sobre los recursos pesqueros (Sidle et al., 1985; Garrity \& Agustin, 1995; Harwood, 1996; Sonak, 2008; Mascarenhas \& Jayakumar, 2008).

Los tsunamis se encuentran entre los más complejos fenómenos físicos responsables de numerosas pérdidas de vidas, y espacio construido en localidades costeras (Wiegel, 1970; Monge 1993; Lagos, 2000; Lagos y Cisternas, 2008). Tal es el caso del terremoto (Mw 9,5) y posterior tsunami de 1960 en el centro sur de Chile. Este evento provocó la destrucción de poblados costeros como Puerto Saavedra, Valdivia y Corral entre otros, generando dos mil víctimas fatales y U $\$ 550$ millones (dólar 1960).

Solo durante la última década del siglo pasado ocurrieron doce eventos de importante magnitud en el mundo; cuyas consecuencias tuvieron un saldo de 4.347 pérdidas de vidas y más de un millón de dólares en daños a la propiedad (ITSU, 1999 en Lagos, 2000). Al respecto, uno de los eventos más importantes lo constituye el caso del 26 de diciembre de 2004, el que generó 283.000 víctimas e importantes daños en propiedades e infraestructura todo el sudeste asiático y el océano Indico, dejando altos costos económicos y sociales (Greenhough et al., 2005; Srinivas \& Nakagawa, 2007; Sonak et al., 2008; Uçklay et al., 2008). En el caso del último terremoto (Mw 8.8) y posterior tsunami el día 27 de febrero de 2010 en el centro-sur Chile, generó más de 400 víctimas fatales y pérdidas materiales en cerca del $17 \%$ del Producto Interno Bruto (PIB), es decir cerca de 30.000 millones de dólares 5 .

Actualmente, en países costeros con una extensa historia de tsunamis como Chile, Japón, Alaska, Canadá, Inglaterra, Australia, Nueva Zelanda e India, los esfuerzos para evaluar los efectos de un tsunami se han orientado hacia la evaluación de la periodicidad, la magnitud y el daño de estos fenómenos. Los principales estudios se han volcado al análisis de registros históricos y geológicos (Dawson et al., 1988; Atwater et al., 1992; Dawson et al., 1995; Dawson et al., 1996; Atwater, 1997; Clague et al., 2000; Lagos, 2000; Lagos \& Cisternas, 2005). Otros estudios han propuesto modelos relacionados con el impacto de la ola en zonas costeras (Dawson et al., 2004; Lagos y Gutiérrez, 2005; Singh, 2007; Madsen \& Fuhrman, 2008), los cuales representan el comportamiento de la inundación de un potencial evento y cuáles serían sus probables consecuencias en una comunidad. Esto se transforma en información de base para el ordenamiento del territorio costero, en el diseño de medidas de mitigación y el manejo de emergencias (Lagos y Cisternas, 2008). Desde el punto de vista social, se presentan estudios asociados al ámbito de la salud sobre la población. Especial atención han recibido los efectos físicos, como fracturas, neumonía, enfermedades producidas por agentes patógenos (Uçklay et al., 2008), y sicológicos, como análisis de la agudización de los estados depresivos y desórdenes en los niveles de estrés (Wickrama \& Kasparb, 2007).

Los estudios relativos a la planificación del territorio de áreas devastadas, como el caso de Sumatra en 2004, han definido los modelos de gestión enfocados en zonas de riesgo y propuestas de vías de evacuación en las costas de India (Sonak et al., 2008; Jaya Kumara et al., 2008; Mascarenhas \& Jayaku-

\footnotetext{
5 Discurso presidencial chileno de 21 de Mayo de 2010.
} 
mar, 2008) o de recuperación de suelos para la actividad agrícola (Tchouaffe \& Tchiadje, 2007). En el caso de Nueva Zelanda, se han desarrollado modelos de gestión incorporados a los instrumentos de planificación, medidas incorporadas (Sidle et al., 2004), y en Chile algunos estudios como MontenegroRomero y Peña-Cortés (2010) han establecido zonas de riesgo de inundación por tsunami como insumo a los soportes de decisión.

El reciente tsunami del 27 de febrero de 2010 en las zonas costeras del centro-sur del país, en Constitución (ciudad costera de la región del Maule), media hora después del terremoto, tres olas de 8,10 y 8 metros respectivamente, devastaron la ciudad. En localidades cercanas como Pichilemu, Pelluhue, Curanipe, Iloca y Coicoi, el mar penetró hasta 200 metros al interior. En el puerto de Talcahuano (Región del Biobío), olas de hasta 5 metros destruyeron prácticamente toda la zona pesquera (Leiva y Quintana, 2010). Sin embargo, el mayor tsunami generado en las costas de Chile durante el último siglo fue el que devastó el centro sur de Chile el 22 de mayo de 1960 . Todos los pueblos costeros entre los $36^{\circ}$ y $44^{\circ}$ grados de latitud sur fueron destruidos o dañados por la acción del tsunami, lo que generó la destrucción de pueblos costeros como Puerto Saavedra, Toltén, Queule, Corral, Bahía Mansa, Quenuir, Maullín y Ancud (Veyl, 1961).

En el caso de la localidad de Corral, la parte baja del poblado fue totalmente destruida. Desde un punto de vista histórico se ha reconocido que el predecesor que rivaliza con las características del tsunami de 1960 fue el tsunami del 16 de diciembre de 1575 (Cisternas et al., 2005). Otro evento, de menor envergadura, ocurrió el 7 de noviembre 1837 (Lomnitz, 1970).

Considerando esta larga historia de tsunamis, la inexistencia de una regularización respecto al riesgo de tsunami en Corral deja expuesta a su población, su infraestructura y el desarrollo de las actividades productivas. En este contexto, la expansión urbana, la falta de planificación y zonificación del riesgo de inundación por tsunami, deja en evidencia la alta exposición de Corral a este tipo de fenó- menos (Montenegro-Romero y Peña-Cortés, 2010).

Ejemplos extremos de consecuencias generadas por tsunamis sobre el uso del territorio en Chile son los dos casos de traslado de poblados costeros completos. El primero ocurrió en Concepción, ciudad que después del tsunami de 1751 fue trasladada $10 \mathrm{~km}$ tierra adentro. El segundo caso corresponde al poblado de Toltén, que después de ser destruido por el tsunami de 1960 fue relocalizado $5 \mathrm{~km}$ tierra adentro, fundando Nueva Toltén. Aparte de estos dos casos, todos los restantes poblados costeros que han sido destruidos por tsunamis históricos se han reconstruido en el mismo lugar. Esta decisión plantea un escenario complejo a la hora de buscar alternativas y nuevos espacios de desarrollo en las zonas costeras (UN Population Division, 2001 en Duxbury \& Dickinson, 2007). Es así como el estudio de la susceptibilidad de inundación por tsunami y repoblamiento de áreas devastadas, es un importante insumo al soporte de decisiones al momento de planificar el desarrollo urbano en localidades costeras. En este contexto, el presente estudio pretende: (1) caracterizar la evolución temporal del repoblamiento y cambio de actividades productivas entre los años 1960 y 2009 en Corral, (2) determinar la percepción del riesgo de inundación por tsunami de sus habitantes $y$, (3) reconocer cómo el riesgo de tsunami está incluido en los Instrumentos de Planificación Territorial (IPTs) de Corral, como base para la planificación y gestión del riesgo.

\section{Materiales y métodos}

\section{Área de estudio}

La localidad de Corral se encuentra emplazada en la comuna del mismo nombre, ubicada en la Región de Los Ríos, a 15 km de Valdivia, la capital regional (Figura $N^{\circ} 1$ ). La comuna tiene una superficie de $766,7 \mathrm{~km}^{2}$ y una población de 5.463 personas. El 67,18\% de la población es urbana, específicamente concentrada en Corral. Coexisten en el espacio urbano $(167,52$ ha), alrededor de cinco actividades entre productiva y residencial, siendo las principales la pesca artesanal e industrial pesquera. 
Figura $\mathrm{N}^{0} 1$

Comuna de Corral Región de Los Ríos

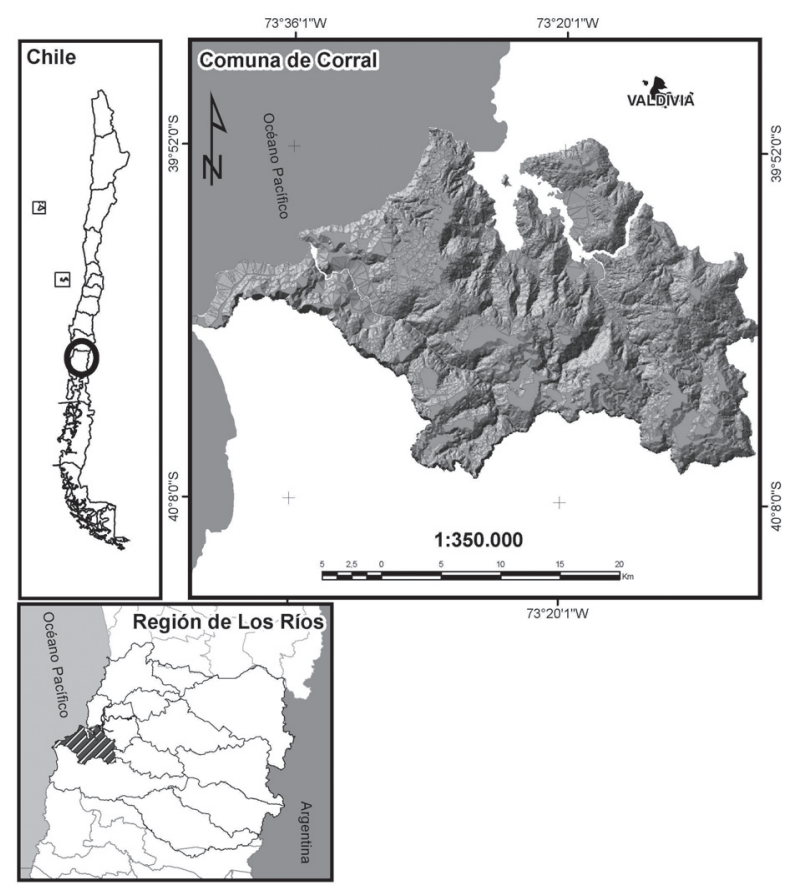

Fuente: Elaboración propia.

\section{Metodología}

Para determinar la superficie afectada por el tsunami y el nivel de inundación alcanzado, se fotointerpretaron fotografías aéreas del año 1960, Vuelo Servicio Aéreo Fotogramétrico (SAF) AER60 distancia focal 163,07 a $80 \mathrm{~mm}$, fotografías $\mathrm{N}^{\circ} 8669, \mathrm{~N}^{\circ} 8666, \mathrm{~N}^{\circ}$ 8667, No 8668 y No 8669 a escala 1:30.000. Estas se georreferenciaron y digitalizaron con el software ArcGIS 9.2. Así se reconocieron aspectos como el tipo de textura en los sectores adyacentes a la línea de la costa, áreas desprovistas de viviendas y la topografía del terreno. Luego, para la identificación del cambio en la localización de actividades residenciales y productivas en las áreas inundadas por el tsunami, se utilizaron fotografías del año 1943 Vuelo Trigonometron N ${ }^{\circ} 55$ IV90 y $N^{\circ}$ 55IV89, escala 1:40.000, y 1982 Vuelo SAF-81 No028084 distancia focal 153,36 UAgII - 3079 a escala 1:60.000, las cuales se georreferenciaron con el software ArcGIS
9.2. La situación actual de la localización de actividades residenciales y productivas en dichas áreas se determinó utilizando fotografías con restitución del vuelo aéreo realizado el 26 de abril de 2009 a escala 1:2.000, para el proyecto "Actualización del Plan regulador Comunal de Corral" estudio ejecutado por la Universidad Católica de Temuco a través del Laboratorio de Planificación Territorial. Sobre estas fotografías se sobrepuso la línea de inundación alcanzada por el tsunami de 1960. Luego se espacializó utilizando el mismo software y se determinó el número de viviendas y actividades productivas al año 2009 que se encuentran en las áreas inundadas por el tsunami de 1960.

Se realizó un cuestionario exploratorio entre octubre y noviembre de 2009 en la localidad de Corral, instrumento que permitió conocer cómo ocurrieron los procesos de repoblamiento y la percepción del riesgo de tsunami por parte de la población. En el 
caso del repoblamiento se reconoció el movimiento de la población entre los años 1960 a 2009 y el cambio de actividad productiva de la población afectada por el tsunami de 1960. En el caso de la percepción del riesgo, el cuestionario se enfocó a conocer la línea de inundación del tsunami de 1960 y el nivel de satisfacción de vivir en una zona de alto riesgo. De acuerdo al INE en el año 2002 (INE, 2002), en la localidad habitaban 507 personas mayores a 60 años de edad, considerando esta cifra y el carácter exploratorio del instrumento y un muestreo no probabilístico, este se aplicó a 23 habitantes, de los cuales 11 fueron mujeres y 12 hombres, nacidos en la localidad de Corral, y que vivenciaron directamente este fenómeno, para ello se localizó un actor clave que permitió localizar al siguiente entrevistado y así sucesivamente de acuerdo al procedimiento "bola de nieve" hasta llegar a una muestra suficiente (Vieytes, 2004). El cuestionario consistió en 6 preguntas abiertas y 15 preguntas dirigidas, las cuales trataron los siguientes temas: límite de inundación por tsunami, repoblamiento de áreas inundadas y cambio en las actividades productivas y percepción actual del riesgo.

Finalmente, para determinar la vinculación del riesgo de inundación por tsunami se realizó una revisión en gabinete de los IPTs en los servicios regionales que licitan y ejecutan estos tipos de instrumentos desde 1960 a 2009: Ilustre Municipalidad de Corral y en el Ministerio de Vivienda y Urbanismo de la Región de Los Ríos.

\section{Evolución de repoblamiento en áreas inundadas por tsunami y cambio en las actividades productivas entre los años 1960 a 2009}

La dinámica de la población, el desarrollo económico y los fenómenos de tsunami, han incidido directamente en la evolución de las actividades residenciales y productivas en las áreas inundadas por el tsunami de 1960. Esto se puede apreciar determinando la línea de inundación por tsunami, que alcanzó una penetración máxima horizontal de 528 m tierra adentro en el sector de Corral Bajo (Figura $\mathrm{N}^{\circ}$ 3 a). A pesar de esto, en el resto de la costa, caracterizada por acantilados de terrazas altas, la penetración fue mucho menor.

Por otro lado, la superficie urbana según el límite urbano vigente desde 1997, es de 167,52 ha, de las cuales el 17,6\% constituye la superficie inundada en 1960. La superficie de mayor concentración de viviendas actualmente abarca 78,5 ha (lo que equivale a un $46,9 \%$ de la superficie total urbana), de esta el $21,1 \%$ se encuentra en las áreas inundadas por el tsunami de 1960. Al respecto, el sector de mayor concentración de viviendas y actividades productivas corresponde al sector

Cuadro $\mathrm{N}^{\circ} 1$

Repoblamiento en áreas inundadas por el tsunami de 1960, localidad de Corral, comuna de Corral.

\begin{tabular}{|l|r|r|r|r|}
\hline SECTOR & $\begin{array}{c}\text { Superficie de sectores localidad } \\
\text { de Corral (ha). Considera solo } \\
\text { concentración de viviendas. }\end{array}$ & $\begin{array}{c}\text { \% de la } \\
\text { superficie total } \\
\text { urbana }\end{array}$ & $\begin{array}{c}\text { Superficie (ha) } \\
\text { inundada por } \\
\text { tsunami de 1960 }\end{array}$ & $\begin{array}{c}\text { \% de la } \\
\text { superficie } \\
\text { inundada por } \\
\text { tsunami de 1960 }\end{array}$ \\
\hline Amargo & 25,3 & 15,1 & 2,3 & 14,2 \\
\hline Corral & 44,5 & 26,6 & 0,0 & 31,8 \\
\hline La Aguada & 8,6 & 5,1 & 16,5 & 0,5 \\
\hline TOTAL & 78,5 & 46,8 & 21,1 \\
\hline
\end{tabular}

Fuente: Elaboración propia. 
de Corral (Corral Bajo y Corral Alto), el cual presenta una superficie de 44,5 ha $(26,6 \%$ de la superficie total urbana), de esta superficie el $31,8 \%(14,2$ ha) se encuentra actualmente repobladas en el área inundada por el tsunami de 1960. En el caso del sector de Amargo solo un $9,1 \%$ de la superficie corresponde a la concentración de viviendas, sector que se encuentra repoblado en las áreas inundadas por el tsunami. En el caso del sector de La Aguada presenta la menor superficie de la concentración de viviendas, ocupando 8,6 ha, lo que representa el 5,1\% de la superficie total urbana y solo el 0,5\% de las áreas inundadas por el tsunami de 1960 (Cuadro No 1 y Figura $\mathrm{N}^{\circ} 2$ ).
En la Figura $\mathrm{N}^{\circ} 2 \mathrm{a}$ ) se observa que antes del tsunami el sector de Corral Bajo estaba ocupado por actividad residencial. En la Figura $\mathrm{N}^{\circ} 2 \mathrm{~b}$ ) se observa el límite del área inundada por el tsunami de 1960. Al año 1982 aparecen viviendas en los cerros, y en el área devastada (Corral bajo) se da cuenta de un nuevo repoblamiento (Figura $\mathrm{N}^{\circ} 2 \mathrm{C}$ ). En ese año las actividades productivas como portuarias, pesqueras artesanales e industriales no se encuentran presentes. En cambio hacia el 2009 se observa que las áreas inundadas por el tsunami de 1960 se encuentran ocupadas principalmente para residencia, es así como en el sector de Corral Bajo se observan 131 viviendas. En cuanto a las actividades

Figura $\mathrm{N}^{\circ} 2$

Repoblamiento de las áreas inundadas por el tsunami de 1960, localidad de Corral. a) año 1942, b) año 1961, c) año 1982 y d) año 2009.

a)

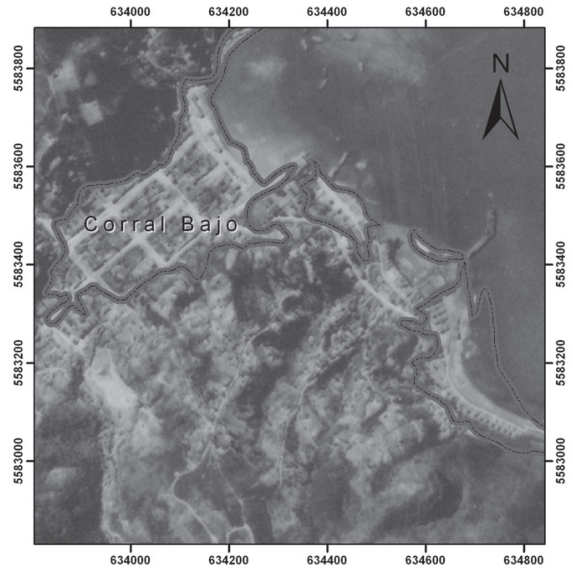

c)

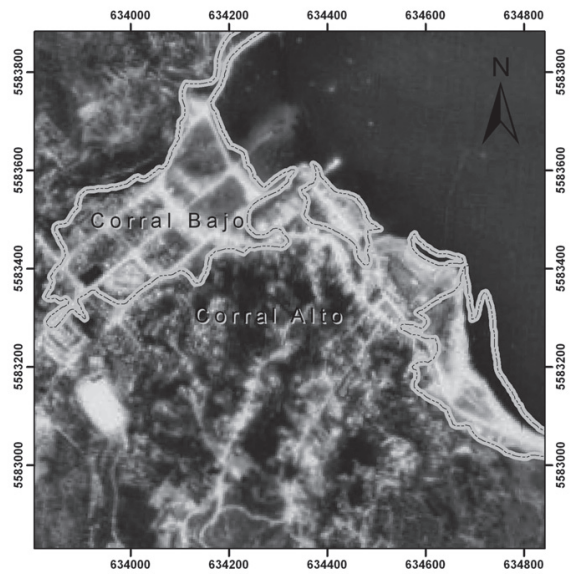

b)

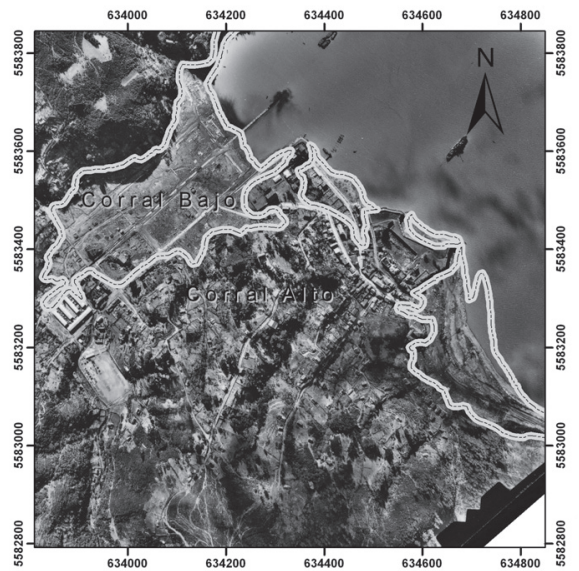

d)

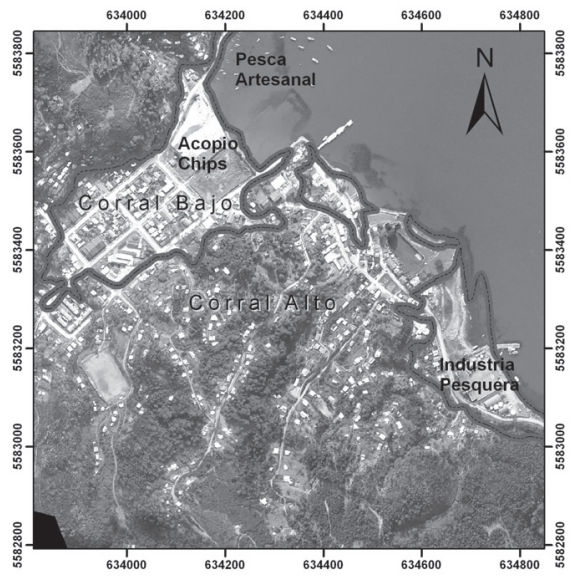

Fuente: Elaboración propia. 
productivas, se reconoce que en el sector de Corral Bajo la actividad portuaria ocupa para el almacenamiento de chips espacios devastados por el tsunami. Así como también lo hacen la, pesca artesanal e industria pesquera (Figura $\mathrm{N}^{\circ} 2 \mathrm{~d}$ ).

Por otro lado, la aplicación del cuestionario permitió validar la línea de inundación fotointerpretada del tsunami de 1960, ya que entregó datos precisos de penetración del tsunami (Figura $\mathrm{N}^{\circ} 3$ ). Se determinó que en Corral Bajo el tsunami llegó hasta la Escuela y cubrió el antiguo teatro (el cual quedó totalmente destruido) ubicado a los pies del Cerro Arica (Figura $N^{\circ} 3 \mathrm{a}$ ). El camino a La Aguada fue cubierto por completo y lo mismo ocurrió con Amargos, sector que fue polo turístico entre los años 1930 y 1960. Allí se encontraba el conocido Hotel Schuster, que fue totalmente destruido por el tsunami (Figura $\mathrm{N}^{\circ} 3 \mathrm{c}$ ).

Al respecto, un aspecto importante en cuanto a la tasa de repoblamiento fue la migración de la población después del tsunami de 1960, tanto dentro como fuera de la localidad. Como muestra el cuadro $N^{\circ} 2$, el $70 \%$ de los entrevistados señaló que cambió de residencia después del tsunami, el 39\% de los entrevistados tuvo pérdida total de sus viviendas.

Un dato de interés obtenido del cuestionario aplicado es que después del tsunami la población que vivía en Corral Bajo se trasladó hacia los sectores más altos. Entre los que destacan el cerro Tacna, Tarapacá y Caupoli-

Figura $\mathrm{N}^{\circ} 3$

Área de inundación de tsunami de 1960 (en rojo), según relatos de los pobladores. de izquierda a derecha imágenes actuales de: a) sector Corral bajo, b) camino a la Aguada y c) Amargos al año 2009.
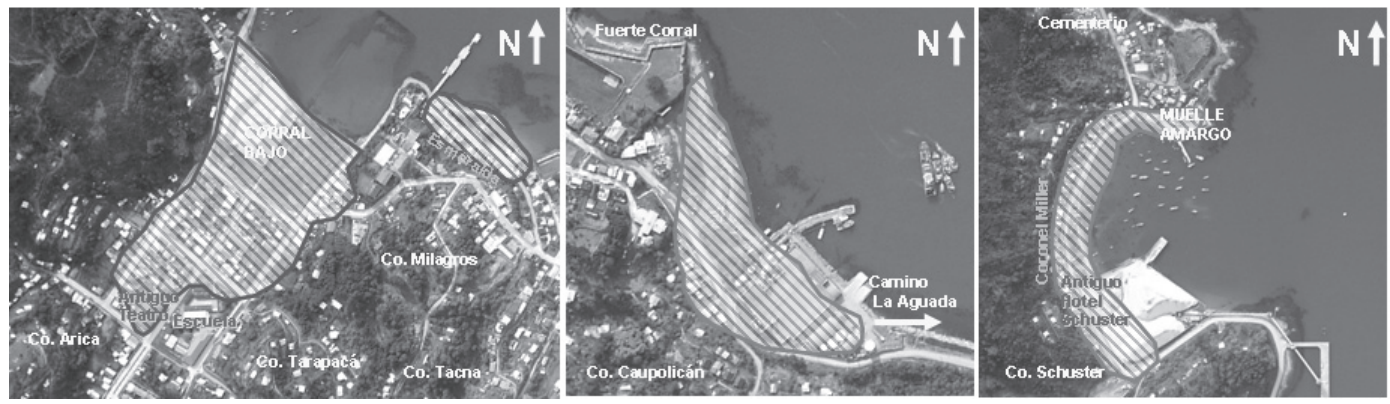

Fuente: Elaboración propia.

\section{Cuadro $\mathrm{N}^{\circ} 2$}

Movimiento de la población según el estado de destrucción de la vivienda entre los años 1960 a 2009.

\begin{tabular}{|c|c|c|c|c|c|}
\hline \multirow{3}{*}{$\begin{array}{l}\text { ¿Su casa en qué condiciones } \\
\text { quedó? }\end{array}$} & \multicolumn{5}{|c|}{ ¿Después del maremoto usted vivió en otro lugar? } \\
\hline & \multicolumn{2}{|c|}{$\mathrm{NO}$} & \multicolumn{2}{|c|}{ Sí } & \multirow{2}{*}{ Total General N% } \\
\hline & $N^{\circ}$ & $\%$ & $N^{\circ}$ & $\%$ & \\
\hline Intacta & 4 & 17,4 & 2 & 8,7 & $6 / 26,1$ \\
\hline Parcialmente destruida & 4 & 17,4 & 3 & 13,0 & $7 / 30,4$ \\
\hline Totalmente destruida & 1 & 4,3 & 9 & 39,1 & $10 / 43,5$ \\
\hline Total general & 9 & 39,1 & 14 & 60,9 & $23 / 100$ \\
\hline
\end{tabular}

Fuente: Elaboración propia. 
cán, este último ubicado en el camino sector de La Aguada. En el caso de Amargos, la población que vivía alrededor del muelle del mismo nombre se reubicó en calle Coronel Miller, la cual se extiende paralela a la playa de Amargos, aunque a mayor altura (Figura $N^{\circ} 3$ ). Es importante señalar que el $17,4 \%$ de los entrevistados que quedó sin sus bienes migró fuera de la región, específicamente a la zona central de Chile (i.e. Valparaíso y Santiago), volviendo a Corral después de 1 a 10 años con ingresos para reconstruir sus viviendas en la misma localidad.

En cuanto a las actividades productivas se detectó el cambio en los rubros de los pobladores después del tsunami de 1960. El Cuadro $\mathrm{N}^{\circ} 3$ indica que el $30 \%$ de los entrevistados era estudiante, actividad que cambió después del tsunami. El resto se distribuyó en actividades como comercio, oficios y pesca artesanal. Asimismo, disminuyen las actividades portuarias e industriales y aparecen actividades como la pesca artesanal, comercio y oficios. Se infiere que el cambio en el rubro de la actividad productiva puede ser producto del traslado de la empresa Siderúrgica a Huachipato (año 1958). Los entrevistados coinciden en que las actividades productivas se concentraban en torno al sector industrial (Siderúrgica Altos Hornos) y servicios asociados a este rubro (puerto-estibadores-buceo de

\section{Cuadro $\mathrm{N}^{\circ} 3$}

Movimiento de rubro laboral de los encuestados entre 1960 a 1962.

\begin{tabular}{|c|c|c|c|c|c|c|c|c|c|c|}
\hline \multirow{3}{*}{$\begin{array}{l}\text { ¿En qué trabajaba usted } \\
\text { antes del maremoto? }\end{array}$} & \multicolumn{5}{|c|}{ Antes del evento de tsunami. } & \multicolumn{5}{|c|}{ Después del evento de tsunami. } \\
\hline & \multicolumn{3}{|c|}{$\begin{array}{l}\text { Rango de edad } \\
\quad \text { (año 1960) }\end{array}$} & \multirow{2}{*}{$\begin{array}{l}\text { Total } \\
\text { general }\end{array}$} & \multirow{2}{*}{$\%$} & \multicolumn{3}{|c|}{$\begin{array}{l}\text { Rango de edad } \\
\quad \text { (año 1960) }\end{array}$} & \multirow{2}{*}{$\begin{array}{l}\text { Total } \\
\text { general }\end{array}$} & \multirow{2}{*}{$\%$} \\
\hline & $\begin{array}{c}10 \mathrm{a} \\
20\end{array}$ & $\begin{array}{c}21 \mathrm{a} \\
30\end{array}$ & $\begin{array}{c}31 \mathrm{a} \\
+ \\
\end{array}$ & & & $\begin{array}{c}10 \mathrm{a} \\
20\end{array}$ & $\begin{array}{c}21 \mathrm{a} \\
30\end{array}$ & $\begin{array}{c}31 \mathrm{a} \\
+ \\
\end{array}$ & & \\
\hline Comercio & & & 1 & 1 & 4,3 & 1 & 1 & 1 & 3 & 13,0 \\
\hline Enfierrador & & 1 & & 1 & 4,3 & & & & 0 & 0 \\
\hline Estibador & & 1 & & 1 & 4,3 & & 1 & & 1 & 4,3 \\
\hline Ganadería/Agricultura & & & & 0 & 0 & & & 1 & 1 & 4,3 \\
\hline Navegante & & & & 0 & 0 & & 1 & & 1 & 4,3 \\
\hline Artesanía & & & & 0 & 0 & & & 1 & 1 & 4,3 \\
\hline Industria & & 1 & & 1 & 4,3 & & & & 0 & 0 \\
\hline Dueña de Casa & & 2 & 1 & 3 & 13,0 & & 2 & & 2 & 8,7 \\
\hline Educación & & 1 & & 1 & 4,3 & & 1 & & 1 & 4,3 \\
\hline Estudiante & 7 & & & 7 & 30,4 & 3 & & & 3 & 13,0 \\
\hline Forestal & & & & 0 & 0 & & 1 & & 1 & 4,3 \\
\hline Sastrería & & & & 0 & 0 & 1 & 1 & & 2 & 8,7 \\
\hline Pesca Artesanal & 1 & 2 & & 3 & 13,0 & 1 & 3 & & 4 & 17,4 \\
\hline Timonel & & 1 & & 1 & 4,3 & & & & 0 & 0 \\
\hline Otros & 1 & 2 & & 3 & 13,0 & & & & 0 & 0 \\
\hline No contesta & & & 1 & 1 & 4,3 & 3 & & & 3 & 13,0 \\
\hline Total general & 9 & 11 & 3 & 23 & 100 & 9 & 11 & 3 & 23 & 100 \\
\hline
\end{tabular}

Fuente: Elaboración propia. 
escafandra). Después del tsunami y traslado definitivo de Altos Hornos se dio un giro hacia la pesca artesanal y comercio.

Percepción de la población en cuanto al nivel de satisfacción de habitabilidad en espacios de riesgo.

Respecto a las condiciones actuales, se reconoció que el $48 \%$ de los entrevistados declara muy altos niveles de insatisfacción de vivir en Corral y que el $35 \%$ se siente protegido frente a un evento de tsunami (Cuadro $N^{\circ} 4$ ). Esto implica que el nivel de insatisfacción no se encuentra asociado a la magnitud ni frecuencia del fenómeno sino que a una sensación de malestar desde el punto de vista económico y de salud, y que la preparación ante un evento de esta envergadura se encuentra asociado más bien a la protección de la integridad física personal que a la sostenibilidad económica.

La inclusión del riesgo de tsunami en los IPTs en la localidad de Corral.

Aunque la comuna de Corral fue oficializada en el año 1925, hacia 1960 no existían
IPTs. Solo al año 1982 se registra el primer Plan de Desarrollo Comunal (1982-1986) y en 1997 el Plan Regulador Comunal de Corral (Cuadro No 5 ).

Actualmente, existen en ejecución 3 IPTs, Plan de Desarrollo Comunal (2008-2012), Plan de Ordenamiento Territorial Comunal (2006) y Plan Regulador Comunal (1997). Los dos primeros consideran el riesgo de tsunami como concepto, aunque lo hace solo de forma indicativa. El único instrumento de planificación territorial de carácter normativo en la comuna y que tiene como función referirse a la zonificación de uso, el Plan Regulador Comunal, no considera el riesgo de tsunami dentro de sus criterios (Cuadro $N^{\circ} 5$ ).

\section{Repoblamiento y cambio de actividades productivas en áreas inundadas por tsunami}

En el sector urbano de la comuna de Corral la superficie de inundación del tsunami de 1960 abarcó 29,5 ha, destruyendo casi la totalidad de Corral Bajo. En ese mismo sector hoy se encuentran 131 viviendas, dejando

Cuadro $\mathrm{N}^{\circ} 4$

Nivel de satisfacción de la población de la localidad de Corral, comuna de Corral.

\begin{tabular}{|c|c|c|c|c|c|c|c|c|}
\hline $\begin{array}{c}\text { ¿Cuál es su nivel de } \\
\text { satisfacción de vivir } \\
\text { en Corral después del } \\
\text { maremoto? }\end{array}$ & Total & $\%$ & \multirow{3}{*}{$\begin{array}{c}\text { ¿Cómo se } \\
\text { siente ante } \\
\text { un nuevo } \\
\text { maremoto? } \\
\begin{array}{c}\text { Muy vulne- } \\
\text { rable }\end{array}\end{array}$} & \multirow{3}{*}{$\begin{array}{r}\text { Total } \\
\\
6\end{array}$} & \multirow{3}{*}{\begin{tabular}{c|}
$\%$ \\
26,1
\end{tabular}} & \multirow{3}{*}{$\begin{array}{c}\text { ¿Está } \\
\text { preparado } \\
\text { para un nuevo } \\
\text { maremoto? }\end{array}$} & \multirow[t]{3}{*}{ Total } & \multirow[t]{3}{*}{$\%$} \\
\hline Muy satisfecho & 5 & 21,7 & & & & & & \\
\hline Insatisfecho & 6 & 26,1 & & & & & & \\
\hline Le es indiferente & 1 & 4,3 & Vulnerable & 5 & 21,7 & $\begin{array}{c}\text { No está pre- } \\
\text { parado }\end{array}$ & 3 & 13,0 \\
\hline Medianamente satisfecho & 4 & 17,4 & $\begin{array}{l}\text { Medianamen- } \\
\text { te vulnerable }\end{array}$ & 2 & 8,7 & $\begin{array}{l}\text { Medianamen- } \\
\text { te preparado }\end{array}$ & 7 & 30,4 \\
\hline Satisfecho & 6 & 26,1 & Protegido & 8 & 34,8 & $\begin{array}{l}\text { Muy prepa- } \\
\text { rado }\end{array}$ & 6 & 26,1 \\
\hline Muy satisfecho & 1 & 4,3 & $\begin{array}{l}\text { Muy prote- } \\
\text { gido }\end{array}$ & 2 & 8,7 & Preparado & 7 & 30,4 \\
\hline Total general & 23 & 100 & Total general & 23 & 100 & Total general & 23 & 100 \\
\hline
\end{tabular}

Fuente: Elaboración propia. 


\section{Cuadro $\mathrm{N}^{\circ} 5$}

Inclusión de la temática de riesgos de inundacion por tsunami (itt) en los Instrumentos de Planificacion Territorial (IPTs) entre los años 1960 a 2009 en la localidad de Corral.

\begin{tabular}{|c|c|c|c|c|c|}
\hline \multirow{2}{*}{$\begin{array}{l}\text { Instrumentos de Planifica- } \\
\text { ción Territorial }\end{array}$} & \multicolumn{5}{|c|}{ AÑO } \\
\hline & $1960-1969$ & $1970-1979$ & $1980-1989$ & $1990-1999$ & $2000-2009$ \\
\hline \multicolumn{6}{|c|}{ Instrumento de Planificación Territorial } \\
\hline $\begin{array}{l}\text { Plan de Ordenamiento } \\
\text { Territorial de Corral }\end{array}$ & & & & & ITT \\
\hline \multicolumn{6}{|c|}{ Instrumento de Planificación Urbana } \\
\hline \multicolumn{6}{|l|}{$\begin{array}{l}\text { Plan Regulador Interco- } \\
\text { munal }\end{array}$} \\
\hline Plan regulador Comunal & & & & ITT & ITT \\
\hline \multicolumn{6}{|l|}{ Plan Seccional } \\
\hline \multicolumn{6}{|c|}{ Instrumento de Planificación Económica } \\
\hline $\begin{array}{l}\text { Plan de Desarrollo Co- } \\
\text { munal }\end{array}$ & & & ITT & ITT & ITT \\
\hline
\end{tabular}

Fuente: Elaboración propia.

totalmente expuestas aproximadamente a más de 524 personas. Aunque este irresponsable comportamiento pareciera ser único de Chile, también se observa en otras latitudes, incluyendo a países desarrollados. En el caso de Italia, el volcán Vesubio soporta en sus faldas una población de 600.000 habitantes, los cuales habitan en áreas devastadas hace dos mil años (Rolandi, 2010). Otro caso icono es Nueva Orleáns, donde la ciudad se vuelve a instalar $3 \mathrm{~m}$ bajo el nivel del mar en el área ya devastada por el huracán Katrina y Rita en el año 2005 (Hori \& Schafer, 2009).

En un evento natural catastrófico también se evidencian procesos de migración parcial o permanente en áreas devastadas. Como fue el caso de Corral, donde la migración ocurrió tanto dentro como fuera especialmente hacia la zona central del país. A diferencia de lo propuesto por Boano (2009), en Corral la migración se dio en forma natural y voluntaria. Esta situación marca una gran diferencia con lo ocurrido en Sri Lanka como consecuencia del tsunami del 2004. Allí, mediante políticas de gobierno, se reubicó la población afectada en "zonas buffer" lejos del lugar donde habitaban originalmente. Se determinó que los segmentos más pobres de la población migra- rán forzadamente. Sin embargo, una pequeña fracción de la población, por inseguridad de perder sus tierras, repobló posteriormnente las áreas devastadas (Boano, 2009).

En cuanto al cambio en las actividades económicas en la localidad de Corral, estas se han visto afectadas por dos hitos o factores históricos importantes: i) el traslado de la siderúrgica de Altos Hornos a Huachipato en 1958 y, ii) el terremoto y tsunami de 1960. Ambos hechos afectaron a la actividad industrial y portuaria, dejando a la localidad con un bajo crecimiento económico. Al respecto, Huang et al. (2008), establece que el grado y período del fenómeno natural está relacionado con el nivel económico de la población antes de la ocurrencia del fenómeno, esto quiere decir que a menor ingreso de la población mayor va a ser el impacto en el desarrollo económico de su territorio.

Actualmente, sectores productivos como portuarios, pesqueros industriales y artesanales, se encuentran localizados en áreas que fueron arrasadas por el tsunami de 1960. Al respecto, la identificación de zonas de alta peligrosidad de inundación por tsunami, tiene gran valor a largo plazo en los planes 
y estrategias de desarrollo, puesto que las inversiones en infraestructura y proyectos quedan mejor resguardadas (Scmidt-Thome et al., 2006). Por otro lado, una de las variables para acelerar la capacidad de resiliencia económica ante un evento natural es establecer "Zonas de viabilidad económica" dentro de un territorio de alto riesgo ante fenómenos naturales, lo que significa un desarrollo económico sostenible para actuales y futuras inversiones (Stewart et al., 2003).

\section{Percepción del fenómeno de la sociedad} civil y marco regulatorio a nivel territorial.

Los altos niveles de insatisfacción de vivir en la localidad de Corral no se relacionan con la percepción del riesgo, sino más bien están relacionados con aspectos de bienestar económico y de salud. Por otro lado, el alto grado de protección y preparación que dice tener la población ante un evento de tsunami conlleva a proponer que la amenaza ante un evento natural está relacionado más bien a la integridad física personal que a la vulnerabilidad y sostenibilidad económica de la población. Tal es el caso del terremoto y posterior tsunami de febrero del 2010 que afectó el Centro-Sur de Chile, en el cual a pesar de haber 400 víctimas las pérdidas económicas alcanzaron US\$30 millones, esto se ajusta a los paradigmas científicos relacionados con la percepción del riesgo. Tal como señala GaiIlard (2008), los individuos eligen incorporar a su vida o no la amenaza de los fenómenos naturales o los peligros. Esto condiciona que una baja percepción del riesgo, sobre todo de los fenómenos inusuales y de alta magnitud, se les perciba como de una baja amenaza. $\mathrm{Al}$ respecto es importante señalar que a pesar de haber vivido el tsunami de 1960 no existe conciencia del riesgo en la población de la localidad de Corral, dejando de lado medidas de prevención que van más allá de la integridad física, es decir medidas de mitigación tanto estructurantes como no estructurantes que disminuyan la vulnerabilidad ante el fenómeno.

Lo anterior se ve potenciado por la inadecuada inclusión del riesgo en los instrumentos de planificación territorial entre los años 1960 y 2010 . Esto ha permitido el desarrollo de las actividades residenciales y productivas en áreas inundadas por el tsunami de 1960 en la localidad de Corral, coartando cualquier medida que disminuya tanto la exposición como la vulnerabilidad ante un evento de similar magnitud. En cuanto a las áreas de alto riesgo de inundación por tsunami en Chile, existen pequeños asentamientos expuestos a la amenaza; son localidades que por su escaso peso relativo en términos demográficos, urbanísticos y/o portuarios sumado a la carencia en la capacidad de gestión de riesgos aún no cuentan con instrumentos que les permitan integrar la amenaza de tsunami en su ordenamiento territorial (Lagos y Gutiérrez, 2005). Ingram et al. (2006) señalan que la capacidad de gestión ante el riesgo se rige según la jerarquía de los asentamientos urbanos, ya que las pequeñas ciudades se encuentran privadas de la influencia política y económica (como el caso de la localidad de Corral) y presentan mayor vulnerabilidad que las megaciudades. Estas últimas no son tan vulnerables a sufrir pérdidas catastróficas ya que se encuentran mejor preparadas tanto en medidas de mitigación estructurantes como no estructurantes ante un fenómeno natural.

Al respecto, los IPTs vienen a ser una solución a la gestión del riesgo de inundación por tsunami. Cabe destacar en este punto la incorporación desde abril de 2011 en el art. 2.1.17 de la Ordenanza General de Urbanismo y Construcción el reconocimiento de las áreas de inundación por tsunami. Allí se define la fase de prevención, desarrollando una zonificación en función y atención a las áreas ya devastadas por un evento de este tipo y considerando de antemano las características de cada asentamiento. En el caso del Plan Regulador Comunal, a pesar de que es el único instrumento de planificación territorial de carácter normativo en la comuna, que entre sus disposiciones tiene como función referirse al uso de suelo o zonificación, no considera el riesgo de tsunami dentro de sus criterios de zonificación.

Andrade et al. (2010) considera que los IPT frente a las nuevas dinámicas de los procesos de cambio en Corral no están lo suficientemente adaptados a la naturaleza y velocidad de las transformaciones que ocurren, en particular las asociadas al desarrollo inmobiliario y a la ocupación industrial, 
como tampoco respecto de las necesidades de protección de las zonas frágiles, a partir de criterios de mayor sensibilidad que los aplicados hasta ahora.

El desarrollo de la gestión ante este tipo de fenómeno en Chile solo se centra en Planes de Emergencia en las localidades que ya han sufrido este fenómeno y cartas de inundación por tsunami generadas por el Servicio Hidrográfico y Oceanográfico de la Armada Chilena. En este contexto Sonak et al. (2008), señalan que el personal del gobierno local y las ONGs que trabajan en las regiones de alto riesgo de inundación por tsunami, se deben implicar en actividades para la construcción de un estado de preparación de las comunidades tanto ante un evento de tsunami, $u$ otros desastres naturales costeros. En la India antes del tsunami de 2004 se carecía de políticas y programas de planificación costera. Sin embargo, hoy allí existe un programa de Manejo Integrado de Áreas Costeras (ICZM). A través de este tipo de instrumentos toma relevancia en la rehabilitación y reconstrucción postsunami, proporcionando una oportunidad para las acciones tanto en áreas devastadas como en el futuro desarrollo de zonas de relocalización de actividades residenciales y productivas a través de la ICZM (Sonak et al., 2008). La capacidad de respuesta y recuperación de áreas devastadas, basadas en el concepto de Manejo Integrado de Zonas Costeras (ICZM), integra las zonas de riesgo de inundación por tsunami a los programas costeros. Sin embargo, estudios de campo sugieren que pocas organizaciones están equipadas para manejar estos programas de recuperación y rehabilitación de una manera organizada (Sonak et al., 2008).

\section{Conclusiones}

Existe un alto riesgo de inundación por tsunami en la localidad de Corral debido a la relocalización de actividades productivas y residenciales en áreas devastadas por el tsunami de 1960. Al respecto, la percepción del riesgo de las personas que sobrevivieron el tsunami de 1960 es baja, lo que queda de manifiesto con la ocupación de los espacios previamente devastados. Lo anterior se asocia a un alto nivel de insatisfacción de vivir en la localidad de Corral producto del escaso acceso a salud y bajo crecimiento económico y no necesariamente a vivir en un lugar con alto riesgo de inundación por tsunami.

En los marcos regulatorios orientados a la planificación del territorio a nivel nacional, ninguno de los IPTs presenta la temática de riesgo de inundación postsunami desde el punto de vista normativo. Queda de manifiesto la falta de conciencia del riesgo en estos instrumentos, evidenciando una importante debilidad en la gestión del riesgo, específicamente en su prevención. De este modo se genera un modelo territorial vulnerable ante la amenaza de un fenómeno natural que casi siempre conlleva resultados catastróficos. Un desarrollo de esta temática en la normativa, y específicamente en la actualización de los Planes Reguladores Comunales, generaría modelos territoriales orientados a la sustentabilidad del territorio.

Finalmente, el estudio de los procesos de repoblamiento y la percepción del riesgo tanto a nivel de Gobierno como de la sociedad civil permiten generar las bases para el trabajo de prevención, capacidad de respuesta y rehabilitación ante el desastre, y desarrollar medidas para la planificación y gestión en un territorio determinado.

\section{Referencias bibliográficas}

ANDRADE, B.; ARENAS F. y LAGOS M. Incorporación de criterios de fragilidad ambiental y riesgo en la planificación territorial de la costa de Chile central. Revista de Geografía Norte Grande, 2010, № 45, p. 5-20.

ATWATER, B.; JIMÉNEZ, N. \& VITA-FINZI C. "Net late Holocene emergence despite earthquakeinduced submergence, south-central Chile". Quaternary International, 1992, № 15-16, p. 77-85.

ATWATER, B. Coastal Evidence for Great Earthquakes in Western Washington. Professional Paper 1560: USGS, 1997, p.77-90.

BOANO, C. Housing anxiety and multiple geographies in post-tsunami Sri Lanka. U.S. National Library of Medicine National Institutes of Health, 2009, Vol. 33, $\mathrm{N}^{\circ} 4$, p.762-785. 
CAICEDO, J. H.; MARTINEGEE, B.; MEYER, H. y REYNA MORENO, J. A. Simulaciones numéricas de propagación de tsunami para la Costa Pacífica de Colombia. Boletín Científico ClOH, 2001, N 19, p. 58-67.

CLAGUE, J.; BOBROWSKY, P. \& HUTCHINSON I. A review of geological records of large tsunamis at Vancouver Island, British Columbia, and implications for hazard. Quaternary Science Reviews, 2000, № 19, p. 849-863.

DAWSON, A.; LONG, D. \& SMITH, D. The Storegga slides: evidence from eastern Scotland for a possible tsunami. Marine Geology, 1988, № 82, p. 271-276.

DAWSON, A.; HINDSON, R.; ANDRADE, C.; FREITAS, C.; PARISH, R. \& BATEMAN, M. Tsunami sedimentation associated with the Lisbon earthquake of 1 November AD 1755: Boca do Rio, Algarve, Portugal. The Holocene, 1995, Vol. 5, No 2, p. 209-215.

DAWSON, A.; SHI, S.; DAWSON, S.; TAKAHASHI, T. \& SHUTO, N. Coastal sedimentation associated with the June 2 nd and 3rd, 1994 tsunami in Rajegwesi, Java. Quaternary Science Reviews, 1996, № 15, p. 901-912.

DAWSON, A.; LOCKETT, P. \& SHI, S. Tsunami hazards in Europe. Environment International, 2004, N³0, p. 577-585.

DUXBURY, J. \& DICKINSON, S. Principles for sustainable governance of the coastal zone: In the context of coastal disasters. Ecological economics, 2007, N63, p. 319-330.

GAILLARD, J. Alternative paradigms of volcanic risk perception: The case of $\mathrm{Mt}$. Pinatubo in the Phillipines. Journal of volcanology and geothermal research, 2008, N¹72, p. 315-328.

GARRITY, D. \& AGUSTIN, P. Historical land use evolution in a tropical acid upland agroecosystem. Agriculture Ecosystems and Environment, 1995, № 53, p. 83-95.

GOBIERNO REGIONAL DE LOS RÍOS. Plan de Ordenamiento Territorial de la Co- muna de Corral, Región de los Ríos. Corral: Gobierno Regional de Los Ríos, 2004.

GREENHOUGH, B.; JAZEEL, T. \& MASSEY, D. The Indian Ocean tsunami: geographical commentaries One year on. The Geographical Journal, 2005, Vol. 171, № 4, p. 369-386.

HARWOOD, R. Development pathways toward sustainable systems following slashand-burn. Agriculture. Ecosystems and Environment, 1996, Vol. 58, N 1, p. 75-86.

HORI, M. \& SCHAFER, M. Social costs of displacement in Louisiana after Hurricanes Katrina and Rita. Population and Environment, 2009, p. 1-23.

HUANG, X.; TAN, H; YANG, T.; ZHOU, J; LI, S. \& LIU, A. Economic loss analysis of flood disaster in Henan Province during 1996-1999. Journal of Natural Disasters, 2008, $\mathrm{N}^{\circ} 17$, p. 31-35.

INGRAM, J.; FRANCO, G.; RUMBAITISDEL RIO, C. \& KHAZAI, B. Post-disaster recovery dilemmas: challenges in balancing shortterm and long-term needs for vulnerability reduction. Environmental science \& policy, 2006, N9, p. $607-613$.

INSTITUTO NACIONAL DE ESTADISTICAS (INE). Chile: Censo de Población y Vivienda 2002. Santiago de Chile: INE, 2002.

JAYA KUMARA, S.; NAIKA, K.; RAMANAMURTHYB, M.V.; ILANGOVANA, D.; GOWTHAMANA, R. \& JENA, B.K. Posttsunami changes in the littoral environment along the southeast coast of India. Journal of Environmental Management, 2008, № 89, p. 35-44.

LAGOS, M. Tsunamis de origen cercano a las costas de Chile. Revista de Geografía Norte Grande, 2000, №27, p. 93-102.

LAGOS, M. y CISTERNAS, M. Depósitos de tsunami como indicadores de riesgo: evidencias sedimentarias. Revista Geográfica de Chile. Terra australis, 2005, N 49, p. 329-351.

LAGOS, M. y GUTIÉRREZ, D. Simulación del tsunami de 1960 en un estuario del cen- 
tro sur de Chile. Revista Geográfica Norte Grande, 2005, №33, p. 5-18.

LAGOS, M. y CISTERNAS, M. Diez años de cambios en el mundo, en la geografía y en las ciencias sociales, 1999-2008. En: $X$ Coloquio Internacional de Geocrítica, 2008, p. $26-30$.

LEIVA, M. y QUINTANA, G. Factores Ambientales y Psicosociales Vinculados a Síntomas de Ataque de Pánico Después del Terremoto y Tsunami del 27 de Febrero de 2010 en la Zona Central de Chile. Terapia psicológica, 2010, Vol. 28, No 2, p. 161167.

LOMNITZ, C. Major earthquakes and tsunamis in Chile during the period 1535 to 1955. Soderdruck aus der Geologischen Rundschau Band, 1970, № 59, p. 938-690.

MADSEN, A. \& FUHRMAN, D. Run-up of tsunamis and long waves in terms of surfsimilarity Coastal. Engineering, 2008, № 55, p. 209-223.

MASCARENHAS, A. Extreme events, intrínsico landforms and human-kind: posttsunami scenario along Nagore-Velankanni coast. Tamil Nadu. Current Science, 2006, № 90, p. 1195-1201.

MASCARENHAS, A. \& JAYAKUMAR, S. An environmental perspective of the post-tsunami scenario along the coast of Tamil Nadu, India: Role of sand dunes and forests. Journal of Environmental Management, 2008, № 89, p. 24-34.

MONGE, J. 1993. Estudios de riesgo de tsunami en costas chilenas. Jornadas chilenas de Sismología en ingeniería antisísmica, 1993, $N^{\circ} 2$, p. 3-22.

MONTENEGRO-ROMERO, T. Y PEÑACORTES, F. Gestión de la emergencia ante eventos de inundación por tsunami en Chile: el caso de Puerto Saavedra. Revista de geografía Norte Grande, 2010, № 47, p. 63-80.

MUNICIPALIDAD DE CORRAL. Plan de Desarrollo Comunal de Corral. Corral: Municipalidad de Corral, 2008.
ROLANDI, G. Volcanic hazard at Vesuvius: An analysis for the revision of the current emergency plan. Journal of Volcanology and Geothermal Research, 2010, N 189, p. 347-362.

SCHMIDT-THOME, P. (editor). Natural and Technological Hazards and Risks in European Regions: Special Paper 42. Espoo: Geological Survey of Finland, 2006a.

SCHMIDT-THOME, P. Integration of natural hazards, risk and climate change into spatial planning practices. Geological Survey of Finland, 2006b, № 193, p. 31-107.

SIDLE, R.; PEARCE, A. \& O'LOUGHLIN, C. Hillslope Stability and Land Use. Washington: American Geophysical Union, 1985.

SIDLE, R.; TAYLORB, D.; LUC, X.; ADGERD, W.; LOWEE, D.; DE LANGEE, W.; NEWNHAMF, R. \& DODSONG, J. Interactions of natural hazards and society in Austral-Asia: evidence in past and recent records. Quaternary International, 2004, № 118-119, p. 181-203.

SINGH, V.S. Impact of the Earthquake and Tsunami of December 26, 2004, on the groundwater regime at Neill Island (south Andaman). Journal of Environmental Management, 2007 (en prensa).

SONAK, S.; PANGAM, P. \& GIRIYAN, A. Green reconstruction of the tsunami-affected areas in India using the integrated coastal zone management concept. Journal of Environmental Management, 2008, $\mathrm{N}^{\circ}$ 89, p. 14-23.

SRINIVAS, H. \& NAKAGAWAB, Y. Environmental implications for disaster preparedness: Lessons Learnt from the Indian Ocean Tsunami. Journal of Environmental Management, 2007 (en prensa).

STEWART, M.G.A.; ROSOWSKY, D.V.B. \& HUANG, Z.C. Hurricane risks and economic viability of strengthened construction. Natural Hazards Review, 2003, № 4, p. 12-19.

TCHOUAFFE, S. \& TCHIADJE N. Strategies to reduce the impact of salt on crops 
(rice, cotton and chili) production: A case study of the tsunami affected area of India. Desalination, 2007, № 206, p. 524-530.

UÇKLAY, A.; SAX, S.; HARBARTH, S.; BERNARD, L. \& PITTET, A. Multi-resistant infections in repatriated patients after natural disasters: lessons learned from the 2004 tsunami for hospital infection control. Journal of Hospital Infection, 2008, №68, p. 1-8.

VIEYTES, R. Metodología de la investigación en organizaciones, mercado y sociedad. Epistemología y técnicas. Buenos Aires: Editorial de las Ciencias, 2004.
VEYL, C. Los sismos y las erupciones de mayo de 1960 en el sur de Chile. Sociedad Chilena de Química, Boletín, 1961, Vol. 11, $\mathrm{N}^{\circ} .1-2$, p. 20-32.

WICKRAMA, A. \& KASPARB, V. Family context of mental health risk in Tsunamiexposed adolescents: Findings from a pilot study in Sri Lanka. Social Science \& Medicine, 2007, Nº64, p. 713-723.

WIEGEL, R. Earthquake engineering. Englewood Cliffs: Prentice-Hall, 1970. 
